\title{
Conception et mise en œuvre de l'interdisciplinarité dans les Observatoires hommes-milieux (OHM, CNRS)
}

\author{
Robert Chenorkian* \\ Interactions hommes-milieux, Aix-Marseille Université, CNRS-INEE, Paris, France
}

Responsable du dispositif des Observatoires hommes-milieux (OHM) qu'il a initié au sein de l'Institut écologie et environnement du CNRS, Robert Chenorkian présente ici les perspectives théoriques et méthodologiques qui ont été mobilisées dans ces expériences menées depuis une dizaine d'années. Le choix interdisciplinaire en est le maître-mot et il est intéressant de saisir comment cette programmation a cherché sa voie pour que les pratiques de recherche qui s'y sont déployées participent à une réactualisation de l'expérience interdisciplinaire. Innovations ou confrontations aux mêmes obstacles des programmes interdisciplinaires du CNRS des années 1980 ? Au lecteur d'en juger et, pourquoi pas, de réagir. Les choix opérés, par conviction ou par tâtonnement, sont situés dans un contexte international, raison pour laquelle $N S S$ en propose une double version, française et anglaise.

La Rédaction

\begin{abstract}
Résumé - Pour répondre à la complexité des crises socioécosystémiques qui affectent de plus en plus une planète anthropisée et globalisée, l'auteur a conçu et mis en œuvre dans le cadre de l'Institut écologie et environnement (INEE) du CNRS depuis 2007 un dispositif global d'interdisciplinarité : les Observatoires hommes-milieux (OHM, 13 existants en 2019), lauréat du PIA1 par un Labex (DRIIHM) depuis 2012. L'auteur présente ce que signifie dans leur cadre l'interdisciplinarité, en quoi elle se distingue des échanges interdisciplinaires classiquement identifiés, comment elle est conçue et mise en œuvre, du plan théorique au plus pratique. Il donne quelques exemples de réalisations et dans une discussion, positionne ce dispositif et ses principes par rapport aux cadres et dispositifs existants.
\end{abstract}

Mots-clés : environnement / développement durable / écosystèmes anthropisés / interdisciplinarité / indisciplinarité

\begin{abstract}
Design and implementation of interdisciplinarity in the Human-Environment Observatories (Observatoires hommes-milieux, OHM, CNRS). Societies are facing social ecosystemic crises which affect the highly anthropized and globalized Planet and which constitute major issues and a huge challenge for the future. Addressing these issues requires facing the very tough complexities of extensively anthropized ecosystems whose understanding demands interaction between the full range of environmental sciences (ESs), i.e. earth, life, human and societal sciences. Interdisciplinarity is not an option, but a structural constraint. The author presents a comprehensive interdisciplinarity facility which he designed within the French CNRS and implements since 2007 to address with this situation: the Human-Environment Observatories (Observatoires hommes-milieux, OHMs). Thirteen of these have been created in France and in other countries in 2019. In 2012 they were the French Governement's PIA1 laureate with the Labex DRIIHM (InterDisciplinary Research Facility on Human-Environment Interactions) for an 8 years funding which has just been renewed for 5 years (2020-2024). The OHMs are dedicated to the study of heavily anthropized sites hit by a "disrupting event" which has upset the ecological, economic and social balances that have developed over decades in the local socio-ecological conditions (for instance, a coalfield mine and its shutdown). In this paper, the author states the qualities needed for this interdisciplinary work in
\end{abstract}

\footnotetext{
خ Cet article est la version française de: «Conception and implementation of interdisciplinarity in the Human-Environment Observatories (OHM, CNRS)», https://doi.org/10.1051/nss/2021003.

*Auteur correspondant : robert. chenorkian@univ-amu.fr
} 
order to deal with these social ecological issues, and specifies the definition of multi-, inter- and transdisciplinarity. He remarks that, within the OHMs, an "enlightened disciplinarity" spontaneously develops between all ESs due to a heuristic open-minded attitude called "indisciplinarity". Three examples of this mode of functioning are presented. They highlight the flexibility and simplicity of the process and its efficacy in developing links among scientists and between these and society in a sustainable development outlook. The OHMs facility provides one of the possibilities to take up the challenge of confronting the hypercomplex ecosytemic crises that arise in highly anthropised socio-ecosystems.

Keywords: environment / sustainable development / human-dominated ecosystems / interdisciplinarity / indisciplinarity

\section{Introduction}

Le changement global fait subir à notre planète une multiplicité de crises socioenvironnementales, souvent très négatives sur le plan local (fermeture d'entreprises, pollutions, etc.) et pour lesquelles la société a besoin de connaissances et de compréhension pour envisager les remèdes appropriés ${ }^{1}$. Ces événements interviennent dans un contexte spécifique, produit de deux faits majeurs qui affectent profondément ce monde qui est le nôtre.

Le premier est l'explosion démographique planétaire, en quantité et en densité, facteur d'artificialisations massives (systèmes anthropoconstruits [Chenorkian, 2014, p. 25-26]). L'homme y est devenu l'acteur prépondérant de tous les systèmes. Le second concerne des évolutions technologiques qui ont permis le développement exponentiel des transports de biens, de personnes et d'idées (Peters et al., 2008), en très grandes quantités, à très longue distance et à très grandes vitesses, voire en quasi-instantanéité (de la conteneurisation aux réseaux sociaux). On peut évoquer la «grande accélération de l'Anthropocène» (Steffen et al., 2015).

La conjonction de ces deux phénomènes a entraîné l'apparition de l' «Anthroposphère », réseau planétaire global qui engendre de manière imprévisible des interactions entre des systèmes disjoints éventuellement très distants. Elle induit ainsi entre ceux-ci l'apparition de rapports qui ne sont plus seulement de hiérarchie ou de panarchie, mais aussi d'an-archie, au sens d'interactions hors de toutes considérations de hiérarchie et de

\footnotetext{
${ }^{1}$ La recherche développée dans ce texte trouve son origine dans des réflexions de l'auteur qui ont conduit à la construction des Observatoires hommes-milieux (OHM, 2007) et du Labex DRIIHM (Dispositif de recherche interdisciplinaire sur les interactions hommes-milieux, 2012) dans le cadre du département EDD (Environnement et développement durable), puis de l'Institut écologie et environnement (INEE) du CNRS. Différents aspects en ont été présentés progressivement lors des séminaires annuels du Labex DRIIHM. Certains ont été abordés dans des publications (Chenorkian, 2014, 2019; Chenorkian et Abbadie, 2017). C'est la première fois que paraît la réflexion dans sa globalité, incluant des aspects jamais exposés.
}

proximité (Chenorkian, 2014, p.24), le tout aux fréquences erratiques de la temporalité et des caractéristiques de l'action humaine. Elle induit une instabilité systémique permanente qui accroît considérablement la complexité de ces écosystèmes que nous qualifions alors d'hypercomplexité ${ }^{2}$.

De ces circonstances découlent deux conséquences majeures. Aucune des sciences de l'environnement (sciences de la Terre, de la vie, de l'homme et de la société - pour peu qu'elles s'en préoccupent-, SDE) ne peut rendre compte à elle seule de cette hypercomplexité et, pour comprendre ces systèmes, il faut i) un abord conjoint et convergent des SDE en interactions et ii) un processus d'étude systémique i.e. qui prenne en considération les objets impliqués et leur organisation, la nature et les dynamiques des interactions qu'ils développent entre eux. Le recours à l'interdisciplinarité n'est donc pas un choix, mais une contrainte structurelle, une absolue nécessité pour parvenir à comprendre ces écosystèmes anthropisés. Ce fait en conditionne les caractéristiques et propriétés. Cette interdisciplinarité doit être toujours disponible (c'est une nécessité), de portée générale (toutes les questions la requièrent), aisée à mettre en place (chaque nouvelle question sollicite plusieurs SDE), très fonctionnelle (qu'elle puisse permettre les interactions quelles que soient les SDE sollicitées), évolutive (capable de se modifier dans sa géométrie et ses questionnements au fur et à mesure de la progression de la compréhension de la question posée et des ajustements qui en résulteraient), pérenne (apte à être disponible en permanence sans réaménagement fondamental ou remise en cause) et résiliente (capable de survivre à la résolution du problème à l'origine de sa sollicitation).

Par ailleurs, dans ces contextes et à l'échelon local ${ }^{3}$ qui nous concerne ici particulièrement, le facteur humain ne peut plus être considéré, ni comme une variable de

\footnotetext{
${ }^{2}$ Au sens où Edgar Morin utilise cette notion pour caractériser le processus d'hominisation auquel il correspond terme à terme (Morin, 1973, p. 130).

3 Aux échelons plus globaux, la complexité des systèmes est telle que, face à l'impossibilité d'en évaluer les conséquences, la question perd un peu de son sens.
} 


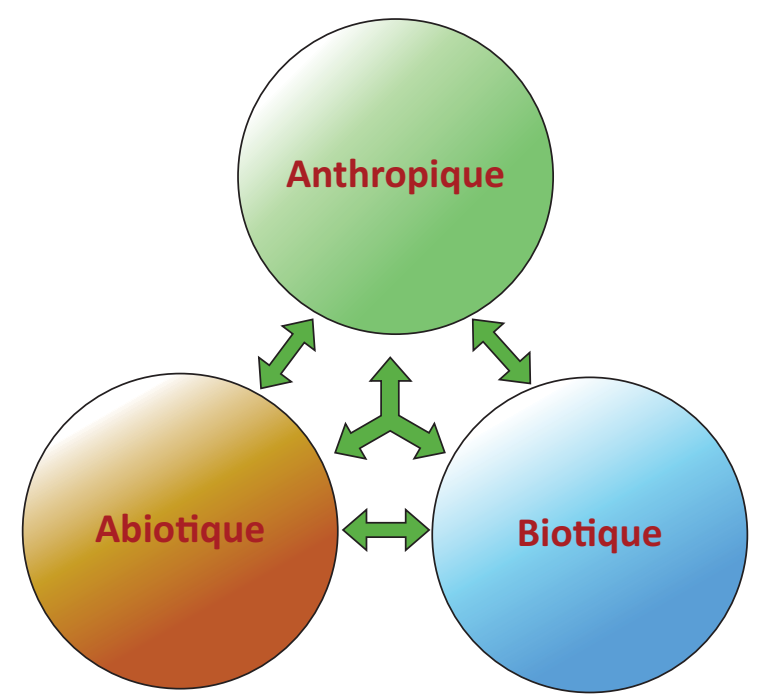

Fig. 1. Écosystème unique (ESU), Single ecosystem (Single ES). Dans les milieux très anthropisés, par la prégnance de l'Anthroposphère, l'anthropique, le biotique et l'abiotique constituent un seul et même écosystème (source: R. Chenorkian).

forçage d'un écosystème naturel, ni comme une composante d'un socioécosystème où un «anthroposystème» interagirait avec un "écosystème » biophysique naturel. Il doit être perçu comme l'une des composantes d'un seul et même écosystème, dans lequel l'anthropique interagit directement avec l'abiotique et le biotique pour constituer ce que nous appellerons un ÉcoSystème Unique $\left(\mathrm{ESU}^{4}\right)$ (Fig. 1). Une telle lecture, heuristiquement déterminante, inscrit l'écologie globale ${ }^{5}$ dans la structure même de l'écosystème et détermine la façon de la mettre en œuvre.

\section{Les OHM}

Pour répondre à ce contexte, aux propriétés des systèmes, aux questions posées et aux attentes de la société, nous avons conçu et mis en œuvre dans le cadre de l'Institut écologie et environnement du CNRS un dispositif dédié: les Observatoires hommes-milieux (OHM). Leur rôle est de permettre, par la convergence des SDE, l'étude des écosystèmes très anthropisés

\footnotetext{
${ }^{4}$ Intégration réclamée pratiquement depuis l'apparition de la notion de socioécosystème par de très nombreux auteurs, mais jamais mise en œuvre (Chenorkian, 2019, note 3).

5 Écologie globale ne signifie pas «écologie de la planète» mais compréhension des écosystèmes grâce à la convergence de toutes les SDE. Si cette notion est celle de notre communauté, les bases qui la sous-tendent étaient déjà évoquées ailleurs il y a plus de 15 ans (cf. Berkes et al., 2003, p. 8: «A complex social-ecological system cannot be captured using a single perspective. It can be best understood by the use of a multiplicity of perspectives »).
}

touchés au premier chef par ces crises socioécosystémiques. Ils ont ainsi été construits pour être des dispositifs d'incitation, de soutien et d'organisation de l'interdisciplinarité requise pour faire face à la très grande complexité de ces phénomènes. Leur démarche, qui inclut aussi une écoute des attentes sociétales, a pour but, au-delà de la mise en œuvre des recherches scientifiques fondamentales et appliquées qui en sont la base, de fournir une compréhension des systèmes afin d'éclairer au mieux les décideurs politiques ou économiques et les participants sociaux au moment de leurs choix. Les OHM positionnent ainsi les chercheurs dans la société.

Les OHM sont issus de trois constats : l'obligation du recours à l'interdisciplinarité pour faire face à la complexité des écosystèmes, surtout très anthropisés, la nécessité d'y impliquer toutes les SDE pour y parvenir, la difficulté permanente et systématique à atteindre un tel résultat.

Tous les $\mathrm{OHM}^{6}$ concernent un lieu anthropisé. Ils sont construits sur un ternaire : un « fait structurant», contexte socioécologique du lieu, un «événement fondateur», qui le perturbe, un «objet focal», produit des deux premiers (Chenorkian, 2014 et 2019). Le fait structurant peut être un bassin sidérurgique et les conséquences écologiques, économiques et sociales qu'induit cette activité, l'événement fondateur, la fermeture des usines, et l'objet focal, le bassin inactivé affecté par cet événement. Toutes les SDE étudient cet objet unique dans une démarche convergente dite d'écologie globale. La recherche dans les OHM fonctionne sur la base d'un appel à projets annuel (de recherche-APR -, doctoral, post-doctoral), de séminaires (de présentation/restitution des recherches pour chaque OHM; à objectif général, sur thématiques ou en transversalités pour le DRIIHM) et d'ateliers spécifiques.

Après plus de douze années d'existence, le projet réalisé, conçu comme un système totalement intégré, va du concept à la mise en œuvre la plus pratique. C'est ce dispositif et ses résultats, en relation avec l'interdisciplinarité requise, que nous présentons ici.

\section{Précisions sémantiques}

Les termes qui décrivent les relations entre les disciplines sont très nombreux : pluri-, multi-, poly-, co-,

6 Il y a aujourd'hui 13 OHM, en France métropolitaine, ultramarine et à l'international: Bassin minier de Provence (2007), Oyapock (Guyane, 2008), Pyrénées - Haut-Vicdessos (2009), Tessékéré (Ferlo, Sénégal, 2009), Estarreja (Portugal, 2010), Vallée du Rhône (2011), Littoral méditerranéen (2012), Nunavik (2012), Pima County (Arizona, États-Unis, 2014), Pays de Bitche (2015), Patagonia-Bahia Exploradores (Chili, 2015), Littoral caraïbe (Guadeloupe, 2016), Fessenheim (2018). 
supra-, méta-, inter-, trans-disciplinarité et probablement d'autres (par exemple Moineau, 2015 ; Kleinpeter, 2013 ; Darbellay, 2011, p. 71 ; Thompson Klein, 2010 ; Godard, 1992, p. 352; Apostel et al., 1972, p. 23-24, etc.). Le ternaire pluri- (ou multi-), inter- et trans-disciplinarité, ordonné selon l'importance des interactions entre les disciplines, est très largement partagé ( $c f$. parmi tant d'autres Piaget, 1972, p. 170; Darbellay, 2018 ou 2011). C'est lui que nous considérerons ici.

Pluri- ou multi-disciplinarité sont très généralement employés pour décrire une coexistence de disciplines sans interaction entre elles. Pour Darbellay (2018, p. 2), la multidisciplinarité est «un processus séquentiel dans lequel des chercheurs de disciplines différentes travaillent, à partir de leur propre point de vue, sur un objet d'étude plus ou moins partagé, de manière indépendante et juxtaposée » ( $c f$. aussi Piaget, 1972, p. 166; Darbellay, 2015, p. 165...). L'objet «plus ou moins partagé » est un fait qui nous rapproche, mais cette situation ne répond pas aux conditions nécessaires évoquées supra.

Le terme «interdisciplinarité» connaît principalement deux acceptions. Pour la première, plusieurs disciplines se combinent pour résoudre un problème (souvent avec la technologie comme médiateur, $c f$. Jollivet, 1992, p. 415 sq. ; Brown et al., 2015). La plupart du temps, la conjonction des disciplines disparaît une fois le problème résolu ( $c f$. Fig. 2.1). Elle ne répond donc que partiellement aux critères recherchés. La seconde est en fait un processus de création de disciplines. Deux disciplines se combinent pour en former une nouvelle (ex: bio-informatique ${ }^{7}$; Fig. 2.2). Cette deuxième acception est très éloignée de nos besoins.

La notion de transdisciplinarité a été définie en 1970 par Jean Piaget. Elle «ne se contenterait pas d'atteindre des interactions ou réciprocités entre recherches spécialisées, mais situerait ces liaisons à l'intérieur d'un système total sans frontières stables entre les disciplines» (1972, p. 170). Elle s'est ensuite développée et diversifiée à partir des années 1990 (McGregor, 2015 ; Thompson Klein, 2010). La définition la plus simple distingue deux approches, l'une dite $\left\langle\right.$ nicolescuian ${ }^{8}$ » et l'autre «zurichoise ${ }^{9}$ » (McGregor, 2015; Max-Neef, 2005 , p. 5). La première conduit à « une nouvelle manière

\footnotetext{
$\overline{7}$ C'est fondamentalement la conception nord-américaine, fruit direct de l'organisation académique et des modes de financement de l'enseignement supérieur et de la recherche aux États-Unis (Graff, 2015 ; nature, special issue, septembre 2015; Frodeman et al., 2010). Cf. aussi la très belle illustration in McPhee et al., 2018, p. 4.

${ }^{8}$ Du nom d'un des fondateurs du CIRET (Centre international de recherches et d'études transdisciplinaires), Bassarab Nicolescu.

${ }^{9}$ Ce nom vient d'un congrès tenu à Zurich en mars 2000 sur la transdisciplinarité (McGregor, 2015 p. 2).
}

de penser le savoir et les questionnements, qui inclut la prise en considération de perspectives éthiques, métaphysiques et même mystiques ${ }^{10}$ » (Bernstein, 2015, p. 6), «elle situe l'homme dans l'Univers. Elle postule que l'économie doit être au service de l'homme. Elle dialogue avec toutes les idéologies humanistes et non totalitaires» (Bourguignon, 2014, p. 4). La «Zurichoise» désigne un «travail dont l'objectif est de concevoir et développer des solutions pratiques aux problèmes du "monde réel" »" (Bernstein, 2015, p. 6; Scholz et Steiner, 2015a). «La transdisciplinarité peut être vue comme une méthode de recherche qui inclut les acteurs politiques, sociaux et économiques, tout comme les citoyens ordinaires, dans le processus de recherche lui-même, dans une perspective de "résolution des problèmes" » (Darbellay, 2015, p. 166; cf. aussi Scholz et Steiner, 2015a, p. 532 et McGregor, 2007, p. 490-491), par la création d'un «nouveau savoir » (McGregor, 2007, p. 487, p. 491-493). Tous les savoirs associés, considérés d'égales importances (Lang et al., 2012, p. 26 sq.; Bernstein, 2015, p. 6 sq.; Scholz et Steiner, 2015a, p. 529), contribuent à la bonne résolution des «wicked problems » auxquels elle se consacre: «justice, durabilité... pauvreté, guerre, génocide, famine, changement climatique, extinction des espèces, épuisement des ressources naturelles et destruction des écosystèmes " (Bernstein, 2015, p. 7 et 11 ; McGregor, 2011, p. 15; McGregor et Donelly, 2014, p. 165 ; Fig. 2.3). Dans les deux cas, conceptions, démarches et objectifs sont très éloignés de ce que nous cherchons ici.

\section{L'interdisciplinarité dans les OHM}

De quelle interdisciplinarité est-il question dans les OHM et le DRIIHM après 12 ans de pratique? Cette question est à examiner sous deux angles : celui de la nature de cette interdisciplinarité et celui de sa pratique.

\section{Une «disciplinarité éclairée »}

Les réponses aux APR sont essentiellement disciplinaires ${ }^{11}$. Chacun œuvre disciplinairement sur un programme propre mais concernant le même objet (focal) et dans le cadre d'une problématisation déjà établie en commun pour tout l'OHM. Cette communauté d'objet dans un cadre partagé dès l'amont permet d'entrer en résonance avec les recherches des autres, de recevoir des éclairages d'autres points de vue, donc aptes à faire ressortir des «topographies» insoupçonnées. Cet éclairement consiste pour chacune des $\mathrm{SDE}$ à prendre

\footnotetext{
10 Toutes les citations anglaises ont été traduites en français par l'auteur.

${ }^{11} \mathrm{cf}$. programmes de recherche lauréats à consulter sur les sites web des OHM. Accès : http://www.driihm.fr/.
} 


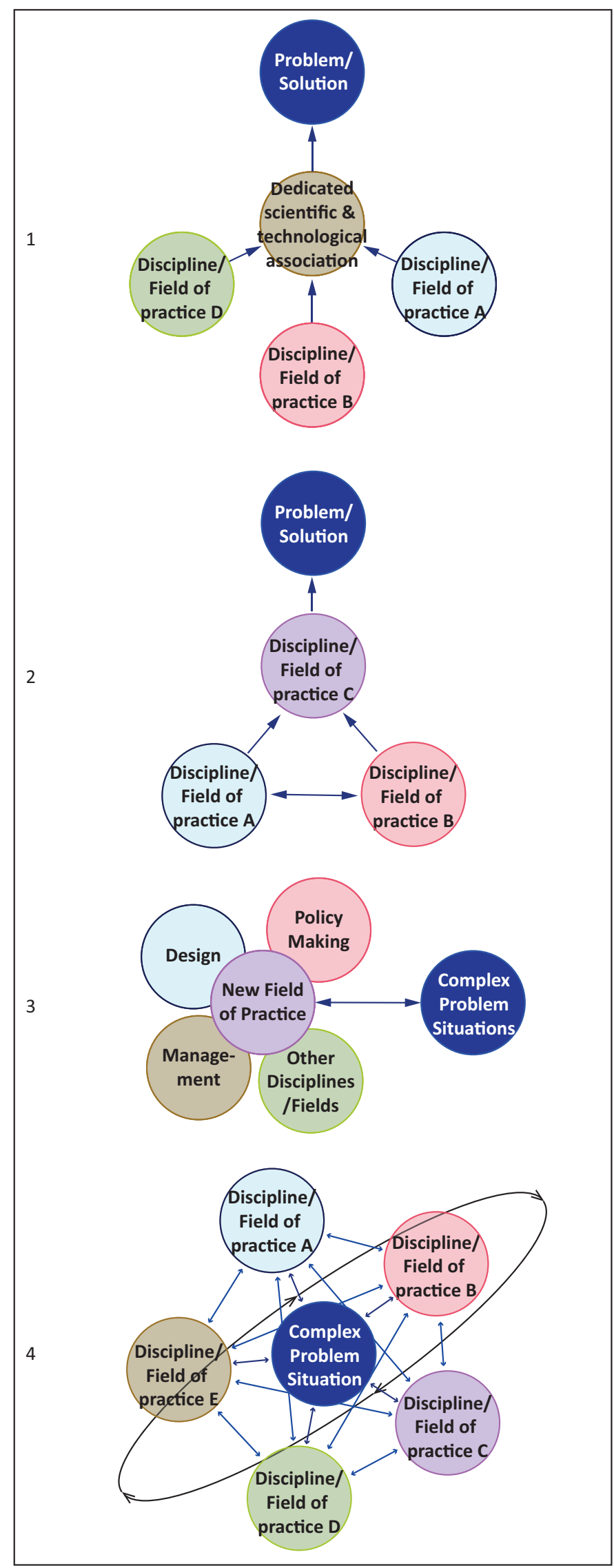

connaissance des résultats des autres en les comprenant bien, de considérer en quoi ils permettent ou nécessitent une reformulation, une réorganisation ou une «reproblématisation» du projet de sa propre recherche disciplinaire, puis, soit d'y revenir pour un nouveau cycle de recherche/communication des résultats/réaménagements du projet, puis/soit d'aboutir à l'étape de modélisation, à la synthèse finale, qui, elle, nécessitera un échange entre les disciplines et leurs résultats pour aboutir à une construction solide et pertinente, intégrant bien toutes les spécificités de chacune d'elles pour un usage sensé et opportun, comme le montrent les exemples infra. L'interdisciplinarité au sens de combinaison circonstancielle de disciplines sur une question (acception 1) peut apparaître, venant porter solution à des cas très particuliers. Mais elle est très rare. La transdisciplinarité, quoi que l'on mette sous ce terme, n’y a jamais été observée.

Nous qualifions la pratique qui se développe dans les OHM de « disciplinarité éclairée » (Fig. 2.4). Elle répond à toutes les exigences énoncées supra. Or, pour qu'elle apparaisse, il faut que chacun soit, dans un esprit d'ouverture, en capacité et en intérêt d'écouter l'autre pour profiter de savoirs différents, ce qui est loin d'être évident.

Depuis le XVIII ${ }^{\mathrm{e}}$ siècle, les disciplines se sont individualisées par différenciation (Moineau, 2015; Bourdieu, 2001). Chacune a donc une méfiance native pour tout mélange qui viendrait affaiblir son identité. En outre, pratiquement tout dans l'organisation actuelle des universités, des organismes de recherche, des instances de recrutement, de l'évaluation de la recherche ou de ses unités, est fondé sur l'excellence disciplinaire, appréciée par des pairs ou experts, qui constituent par nature des instances de contrôle de la bonne conformité à la norme dominante de la discipline engagée. Aussi, si l'appel à l'interdisciplinarité est omniprésent et, semble-t-il, très

Fig. 2. Modes de relation entre les disciplines. 1 et $2-$ Interdisciplinarité : 1- plusieurs disciplines convergent pour la résolution d'une question précise; 2- deux disciplines s'hybrident pour en former une troisième en un processus de disciplinarisation. 3- Transdisciplinarité : le processus de recherche associe dans une même démarche, scientifiques, politiques, économistes, etc., pour construire dans un mode de relation différent un "nouveau savoir». 4- "Disciplinarité éclairée »: toutes les disciplines étudient le même objet et agissent en considérant et intégrant directement les apports des autres, en se nourrissant de ces échanges par itération autant que nécessaire pour proposer une compréhension globale du système complexe étudié (sources : 1 et 4 , Chenorkian; 2 et 3 , McPhee et al., 2018, p. 4). Toutes les illustrations appliquent les codes de McPhee et al., 2018, p. 4. 

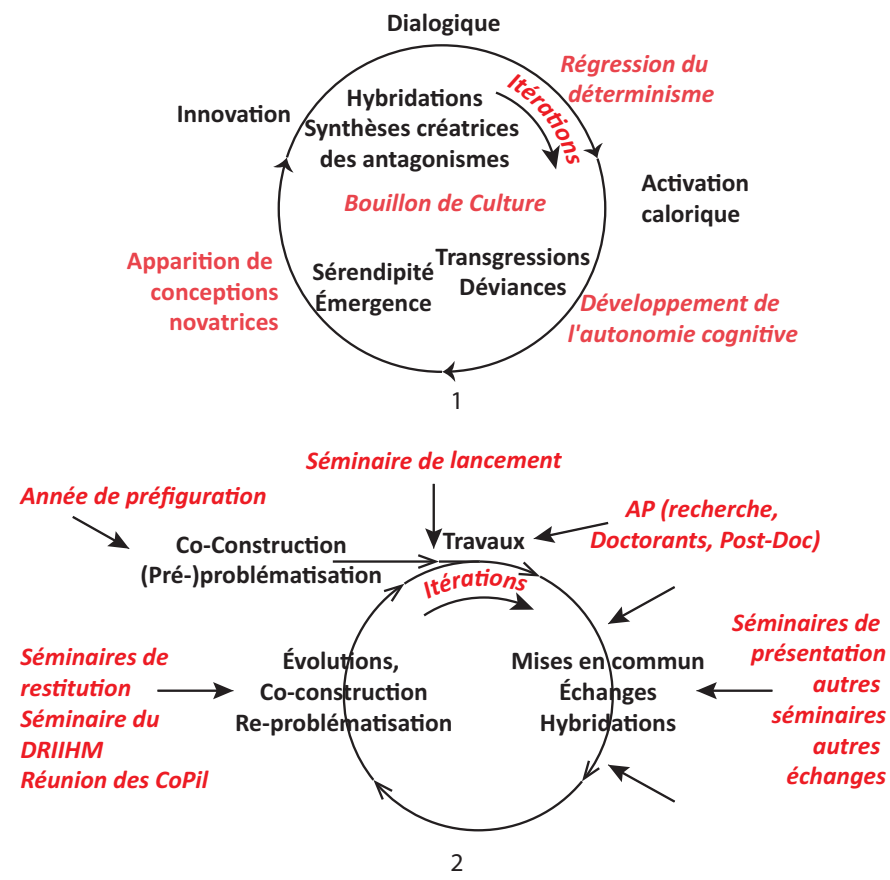

Fig. 3. Le bouillon de culture selon Edgar Morin et sa constitution dans les OHM. 1. Le développement d'échanges dialogiques permet, par l' « activation calorique », un affaiblissement des déterminismes, qui rend possible transgressions et déviances. Des rencontres improbables peuvent ainsi intervenir, favorisant sérendipité, émergence et innovation, avec hybridation et synthèses des contraires, situations éminemment dialogiques, etc. Ce schéma issu des Idées a été directement appliqué au mode de fonctionnement des OHM, terme à terme (2) par la mise en place des modes de fonctionnement présentés (mixités, occasions de rencontres spécifiques, attitudes lors des échanges, etc.). Pour les OHM, ce cercle est fondé sur les activités annuelles (source: R. Chenorkian).

consensuel $^{12}$, les barrières disciplinaires sont toujours bien présentes et très fortes.

L'interdisciplinarité demeure toujours le fruit d'une action volontariste, fondamentalement transgressive (Bourdieu, 2001, p. 87 ; Thompson Klein, 2015, p. 11 sq.) et intrinsèquement génératrice de réactions et conséquences négatives. La «disciplinarité éclairée» et ses échanges actifs et pérennes entre les disciplines n'apparaîtront donc pas spontanément (Bourdieu, 2001, p. 73).

Pour atteindre ce but, nous avons choisi de ne pas poser de prérequis (pas d'interdisciplinarité demandée a priori, pas de recherche préalable d'un langage commun $^{13}$ ) qui sont tous des facteurs d'échec, mais au

\footnotetext{
12 Ce n'est pas nécessairement vrai pour les Anglo-Saxons (Jacobs et Frickel, 2009).

13 Si un tel but était atteint, la démarche serait alors de disciplinarisation, car à langage unique, discipline unique.
}

contraire de promouvoir un esprit d'ouverture, en faisant appel à ce qui est tout à la fois une attitude et un outil : l'indisciplinarité.

Ce terme est d'un usage relativement commun dans la littérature anglo-saxonne et particulièrement nord-américaine. Indisciplinaire ou indisciplinarité indiquent le plus souvent l'absence de discipline (ce serait donc plutôt 《a-disciplinaire », voire « dédiscipliné », $c f$. Darbellay, 2015, 2016), comme ce que l'on retrouve par exemple dans les formations undergraduate des États-Unis qui se caractérisent par l'absence de formation disciplinaire ( $c f$. notamment Tribe, 1997 ; Norman, 2006). Pour d'autres, il peut signifier anti-disciplinarité, attitude volontariste contre les disciplines et pour leur suppression (Jacobs, 2013, p. 123 ; Darbellay, 2016, p. 363).

L'indisciplinarité que nous requerrons ici est totalement différente. C'est une posture heuristique d'ouverture, apte à faciliter les relations entre les disciplines (Loty, 2005; Catellin et Loty, 2013). Ce n'est pas aux disciplines qu'elle s'attaque, mais aux constructions étanches (« en silo », Jacobs, 2013, p. 17), aux barrières qui séparent les champs disciplinaires et qu'elle a pour vocation d'affaiblir. Elle favorise les échanges pour des mobilités intra- et inter-disciplinaires. Elle permet ainsi de conserver des démarches toujours disciplinaires, mais ouvertes, offrant des possibilités de mouvements, d'échanges et d'évolution fertiles. La nécessité de réduire les barrières pour permettre l'interdisciplinarité avait bien été soulignée par Darbellay en 2011 (p. 84 ${ }^{14}$ ). Pour nous, l'indisciplinarité ainsi conçue est la clé de cette « disciplinarité éclairée ». « Disciplinarité éclairée » et indisciplinarité nous permettent de mettre en œuvre l'inter-disciplinarité dont nous avons besoin.

La question est maintenant celle de la mise en œuvre de cette pratique, dont on a constaté qu'elle est devenue usuelle dans les OHM.

\section{De la théorie à la pratique}

La solution adoptée dans les OHM et le DRIIHM est fondée sur les principes développés par Morin, dans Les idées, t. 4 de La méthode (1991, p.39-49), pour

14 «Au lieu de prétendre à une théorie unifiée de l'interdisciplinarité [...] il apparaît qu'il faille en premier lieu créer une culture de tolérance réciproque entre les disciplines, une forme d'empathie (inter-)disciplinaire au sens cognitif de Berthoz, c'est-à-dire la capacité à comprendre le point de vue de l'autre, à se mettre à sa place, mais sans renoncer pour autant à son identité. Il s'agit d'opérer un décentrement qui permette de manipuler plusieurs points de vue complémentaires dans le traitement de l'homme et du social compris dans leur complexité ». 
l'affaiblissement des déterminismes culturels afin de libérer la pensée et la connaissance par les échanges dialogiques, la «chaleur » culturelle et le développement d'un «bouillon de culture» (Fig. 3).

Le processus débute par l'ouverture d'une activité d'échanges dialogiques qui vise à entraîner une régression des déterminismes, des modes de pensée convenus et normés. Cette ouverture permet le développement de «chaleur culturelle, d'activation calorique [...] d'échanges, débats animés, d'antagonismes. Cette activation comporte [...] beaucoup de déperdition d'énergie et [...] d'aléas, mais [...] apporte des chances multiples à l'essor des idées et des connaissances » (Morin, 1991, p. 39-49). Il en découle des comportements de transgression, l'ouverture d'espaces de liberté aux pensées discordantes qui permet l'autonomie cognitive. Cette capacité nouvelle permettra l'expression de déviances qui pourront «susciter une hybridation ou, mieux, une synthèse créatrice entre les idées contraires » (Morin, 1991, p. 39-49). Cet abaissement des barrières va favoriser l'apparition de nouveaux échanges dialogiques. La boucle est bouclée, le cercle vertueux réalisé, les itérations peuvent s'enchaîner, créant ainsi le milieu vivant du «bouillon de culture».

Nous avons directement appliqué ce processus à notre question: comment soutenir le comportement transgressif du passage des barrières disciplinaires et favoriser celui-ci en les affaiblissant, favoriser alors la capacité éminemment subversive d'imprévoir l'attendu, pour reconnaître l'inattendu et découvrir l'inconnu.

Il s'agit, d'une part, d'évacuer autant que possible les facteurs de blocages. C'est probablement l'étape la plus transgressive car elle se heurte aux pratiques habituelles et aux systèmes établis. Tout d'abord proscrire tout ce qui disciplinarise, ce qui fait obstacle aux «échanges dialogiques » et obture les voies nouvelles. En premier lieu, c'est exclure non pas l'expertise mais la posture d'expert, qui interdit tout débat. Deuxièmement, c'est voir dans l'excellence requise, non pas une opportunité de reconnaissance par une communauté mais son intérêt pour le travail à faire. Troisièmement, c'est éviter de chercher systématiquement le consensus dans l'échange, ce qui neutralise en chacun et collectivement toute capacité à transgresser.

D'autre part, c'est favoriser ce qui ouvre, décloisonne et provoque le débat ouvert. C'est développer la mixité dans tous les domaines, qu'il s'agisse des cultures scientifiques et des disciplines, des populations dans leurs fonctions ou niveaux (des étudiants aux chercheurs seniors). C'est faire coexister, cofonctionner cette diversité et, pour cela, lui aménager des lieux et des temps de rencontres selon ces principes, afin que chacun puisse/doive parler, écouter, échanger dans des cadres tant formels qu'informels.
La mise en place d'un OHM est un processus de coconstruction. Une fois les trois bases bien posées ( $c f$. supra), il débute par l'identification des premières thématiques par lesquelles pourra être abordée l'hypercomplexité de l'ESU. Cette étape de "pré-problématisation », essentielle, permettra de construire un dispositif qui fera sens pour tous et que chacun pourra s'approprier. C'est là que se joue la capacité à faire converger les disciplines sur l'objet commun en construisant sa potentielle attractivité pour «toutes» les SDE.

La première année d'un OHM, dite de préfiguration, est consacrée à ce travail. Réunions, colloques, ateliers sont organisés, dont certains ouverts à la société (monde politique, économique, associatif, simples citoyens) de manière à permettre aussi à ce moment de préparer pour l'avenir les possibilités d'appropriation et d'échanges. Cette coconstruction sociétale permet d'établir les liens qui donneront efficacité aux retours des travaux vers la société. Cette première année se conclut par un séminaire de lancement où les résultats de toutes ces réflexions sont présentés aux communautés concernées.

La mise en pratique de la "disciplinarité éclairée » peut ensuite se décrire en 4 étapes.

\section{Construire un réceptacle (un « réacteur») et créer le bouillon de culture}

C'est le Labex DRIIHM qui le constitue, à travers toutes ses composantes (OHM, séminaires de lancement et de restitution, séminaires annuels du Labex, APR et AP doctorants et post-doctorants, ateliers thématiques, groupes de travail, initiatives transversales). C'est tout ce qui, dans le Labex DRIIHM, occasionne ou facilite les échanges et construit la communauté.

Le bouillon de culture est constitué de ce qui entraîne mixité et interactions : coconstruction, (pré-)problématisation et reproblématisation, participation à tous les séminaires. C'est aussi penser/organiser la mixité scientifique et sociale et l'ouverture des échanges. Ces coconstructions concernent les chercheurs de toutes les SDE. Certaines étapes doivent aussi être ouvertes à toute la société, pour bien prendre en compte les attentes sociétales ${ }^{15}$.

\section{Identifier et associer les ingrédients}

La construction de cette mixité et de tout ce qui va venir nourrir ces occasions d'interventions et d'échanges passe par l'implication de toutes les SDE et de tous les lauréats d'un projet de recherche. Les séminaires intègrent tous les membres du DRIIHM : chercheurs, praticiens, doctorants et post-doctorants, étudiants en

\footnotetext{
${ }^{15} \mathrm{NB}$ : il ne s'agit en aucun cas de transdisciplinarité, $c f$. infra, Discussion.
} 
master, membres de toutes les instances du dispositif $(\mathrm{CD}, \mathrm{CS}, \mathrm{COS}, \mathrm{CoPil} \mathrm{OHM})$; une ouverture est ménagée à tous les partenaires: les universités, les EPST, les entreprises, les associations, voire les particuliers.

\section{Apporter et entretenir les énergies}

Cette étape passe par l'entretien permanent et par tous de l'affaiblissement des barrières et de l'ouverture dialogique; elle veille à ce que le système ne retombe pas dans les ornières profondes des anciennes habitudes; elle veille à la «bonne» tenue de toutes les réunions et de la posture des participants, à l'hétérogénéité permanente, elle lutte contre la redisciplinarisation sournoise, résiste à la tendance au consensus. Elle accepte aussi la coexistence entre organisation et inorganisation (partielle), le temps perdu et parfois retrouvé par des accélérations fertiles, tout cela étant des conséquences naturelles du système et du bouillonnement qu'il doit provoquer.

\section{Laisser s'opérer les échanges et les relancer}

Cette étape donne avant tout du temps aux séminaires (3 jours pour celui du DRIIHM) comme aux moments de convivialité. L'intervention directe pour construire l'interdisciplinarité ne fonctionnant pas, il importe, par une action indirecte, de favoriser et laisser évoluer, tout en étant très directif sur le cadre et les principes et en veillant à la fluidité du milieu et aux bonnes possibilités de mobilités. Les sessions de séminaires fonctionnent en acceptant localement l'inorganisation, voire le détournement, en laissant la liberté des échanges et des pensées, en donnant du temps d'amorçage, mais en étant strict sur les horaires. L'organisation de longs temps de pause en des lieux ouverts non contraints favorise les mouvements et les échanges imprévus.

L'équilibre d'une démarche qui cherche à associer les contraires (ordre et désordre, hiérarchie et anarchie, rigueur et souplesse) est instable et ne peut apparaître que dans le mouvement. C'est donc une réitération permanente, un cercle sans cesse renouvelé qu'il faut entretenir. C'est le Labex DRIIHM qui joue ce rôle à travers toutes ses composantes (OHM, séminaires, AP, etc.). Il construit la communauté.

\section{Exemples de «disciplinarité éclairée » telle qu'elle prévaut dans les OHM}

\section{OHM Pyrénées - Haut-Vicdessos: du mercure marin dans les lacs des Pyrénées}

La vallée du Haut-Vicdessos (Encadré 1) a connu une grande activité minière depuis la plus haute Antiquité.
Une série de carottages a été entreprise dans des lacs pour $\mathrm{y}$ trouver des traces de pollution au plomb. C'est du mercure qui a été trouvé, avec une signature isotopique marine. Les sociétés de pêche pratiquent l'alevinage des lacs depuis des décennies pour des usages récréatifs. Des recherches ont été menées auprès des sociétés de pêche, des piscicultures productrices et dans leurs archives. La présence de mercure est due au passage dans les années 1960 d'une alimentation provenant d'animaux terrestres aux farines de poissons, faites des sous-produits de poissons pêchés à proximité des grands fleuves fortement pollués au mercure. Les auteurs formulent l'hypothèse qu'aujourd'hui environ une tonne par an de mercure marin se retrouverait ainsi à terre en France.

Encadré 1. OHM Pyrénées-Haut-Vicdessos : du mercure marin dans les lacs des Pyrénées.

Disciplines impliquées : paléoenvironnement, sédimentologie, géochimie isotopique, enquêtes sociales, travail en archive, histoire

Discipline de publication: géochimie

Pour en savoir plus : voir la publication initiale, Hansson S.V., Sonke J., Galop D., Bareille G., Jean S., Le Roux G., 2017. Transfer of marine mercury to mountain lakes, Scientific Reports, 7, 12719, https://doi.org/10.1038/ s41598-017-13001-2.

Au-delà du local, comment stopper cette pollution, l'arrêt de l'alevinage n'étant ni écologiquement ni socialement acceptable (eutrophisation catastrophique des lacs)?

\section{OHM Littoral méditerranéen : contamination des eaux de baignade par les produits anti-UV à Marseille}

Cet OHM a identifié la thématique «Les fréquentations touristiques et récréatives des milieux côtiers » comme l'un de ses deux objectifs de recherche (Encadré 2). Des recherches concernent les plages marseillaises du Prophète, de la Lave et de la Pointe - Rouge.

Une première recherche de géographie-sociologie a porté sur les usages des plages (qui, quand, comment, où). Cette première description a permis de contextualiser une interrogation sur les contaminations par les produits anti-UV engageant deux actions: dosages chimiques des eaux de mer et usages des crèmes solaires (quels anti-UV - minéraux ou biologiques - fréquences et modes d'utilisation, âge des utilisateurs, etc.). Le fait de pouvoir bénéficier de ce premier cadre (types de populations, effectifs, heures de fréquentations, pratiques de baignades) a permis d'effectuer d'emblée des 
Encadré 2. OHM Littoral méditerranéen : contamination des eaux de baignade par les produits anti-UV à Marseille.

Disciplines impliquées : géographie, sociologie, hydrologie, géochimie, chimie de l'environnement

Discipline de communication: géochimie

Une première communication, présentée à la Goldschmidt geochemistry conference de Boston en 2018, axée sur la contamination par le dioxyde de titane $\left(\mathrm{TiO}_{2}\right)$ a connu une grande couverture médiatique (une dépêche reprise 72 fois par des journaux de différents pays).

Pour en savoir plus : voir le résumé de la communication, Labille J., Slomberg D., Catalano R., Robert S., Boudenne J.-L., Apers-Tremelo M.-L., Masion A., Garidel C., 2018. Evaluation of the environmental exposure to nanoparticulate UV-filters used in sunscreens. Communication au Goldschmidt Boston 2018, 12-17 août, Boston, https://www.researchgate.net/publi cation/327974623_Evaluation_of the_environmenta 1_exposure_to_nanoparticulate_UV-filters_used_in_sun screens.

prélèvements d'échantillons d'eau géo- et chronolocalisés hautement pertinents et d'obtenir des résultats extrêmement significatifs.

Ainsi, $80 \%$ des usagers se baignent. Ils s'enduisent de crème solaire 2,7 fois en moyenne par sessions et autant avant qu'après la baignade. Les pics de concentration de produits anti-UV apparaissent à 16 heures, à $2 \mathrm{~m}$ de distance du littoral et $40 \mathrm{~cm}$ de profondeur. En première approximation, $68 \mathrm{~kg} /$ jour de produits anti-UV seraient relargués dans les eaux de la plage du Prophète en haute saison, soit 5,7 t pour la saison. Pour les $\mathrm{TIO}_{2}$, l'évaluation est de $340 \mathrm{~g} /$ jour soit $71 \mathrm{~kg} /$ mois.

Au-delà du local, quel est l'impact global de ce relargage? Comment interrompre une source considérable de pollution côtière par contact ou via les réseaux trophiques?

\section{OHM Pyrénées - Haut-Vicdessos: utilisation des orri des Pyrénées, paléoenvironnement, biodiversité}

La question initialement posée était celle du poids des activités pastorales sur la diversité floristique des espaces d'altitude (Encadré 3). La reconstitution de la paléovégétation/paléodiversité floristique a été obtenue par une étude palynologique à haute résolution temporelle d'enregistrements sédimentaires (tourbières et lacs) datée par les méthodes $\mathrm{du}{ }^{14} \mathrm{C}$ ou des radionucléides $\left({ }^{137} \mathrm{Cs}\right.$ et $\left.{ }^{210} \mathrm{~Pb}\right)$. Toute la difficulté était de préciser des charges pastorales sur plus d'un siècle, pour potentialiser les reconstitutions paléoenvironnementales.

Encadré 3. OHM Pyrénées-Haut-Vicdessos : utilisation des orri des Pyrénées, paléoenvironnement, biodiversité.

Disciplines impliquées : paléoenvironnement, sédimentologie, géochimie isotopique, exploitation d'archives, enquêtes sociales

Discipline de publication : paléoenvironnement

Pour en savoir plus : voir la publication initiale, Galop D., Houet T., Mazier F., Leroux G., Rius D., 2011. Grazing activities and biodiversity history in the Pyrenees: new insights on high altitude ecosystems in the framework of a Human-Environment Observatory, PAGES (Past Global Changes) News, 19, 2, 53-55, https://doi.org/10.22498/pages.19.2.53.

La transhumance estivale dans les Pyrénées suppose la montée des bêtes et des familles sur les zones d'estives dans la vallée où ont été construites des orri, maisons familiales en pierres sèches - chaque famille en possédait plusieurs - où étaient abrités hommes et bétails pendant tout l'été. Lors de la montée, l'administration forestière prélevait des taxes et la douane s'assurait de l'absence de contrebande avec l'Espagne. Cela a généré d'abondantes archives, entièrement dépouillées, et l'on connaît aujourd'hui, par année, le nombre d'animaux montant et descendant, par famille, et leur destination. Une enquête auprès des derniers bergers a relié sur le terrain orris et familles. Le croisement de ces informations a permis de reconstituer avec précision les fluctuations des charges pastorales à l'échelle de chaque orri ou éleveur, par année ; ces informations géo- et chrono-localisées ont pu être confrontées aux résultats des études paléoenvironnementales. Cela a notamment mis en évidence l'existence d'une relation positive entre charge pastorale et diversité floristique. Les résultats montrent clairement l'effondrement de cette dernière, proportionnellement à la réduction des troupeaux et à l'enfrichement/ landification.

Ces résultats révèlent l'intérêt de revitaliser l'activité pastorale avec des systèmes extensifs si l'on veut assurer le maintien d'une biodiversité (par effet cascade, plantes, insectes, amphibiens, oiseaux, etc.).

Au-delà du local, ces résultats contredisent une idée reçue selon laquelle l'activité humaine réduit toujours la biodiversité et qu'une politique de préservation ne peut être que nationale. Leur prise en compte sera donc essentielle pour la bonne conception d'un soutien à la biodiversité. 


\section{Discussion}

L'interdisciplinarité qui vient d'être illustrée s'inscrit bien évidemment dans la grande tendance de rapprochement fonctionnel des disciplines ou dans la démarche de prise en compte des partenaires non acteurs de recherche qui se développe depuis des décennies. Elle s'en distingue pourtant fondamentalement pour ce qui est des besoins identifiés, de la façon de répondre à la complexité des ESU, de la manière de prendre en compte les besoins de la société et de partager avec elle les connaissances acquises sur ces crises socioécosystémiques.

La pratique de l'interdisciplinarité dans les OHM pourrait paraître très proche de la définition de F. Darbellay $(2018$, p. 2) : «Des chercheurs travaillent ensemble, à partir de - et entre - leurs points de vue disciplinaires, sur un objet d'étude commun, de manière coordonnée, interactive et dans une visée intégrative ». Elle s'en différencie cependant sur des points essentiels, principalement liés à l'organisation du travail et à ses temporalités. Comme on l'a vu, les chercheurs travaillent ensemble en vue d'une coconstruction de l'objet d'étude et de sa problématisation qui permettent d'aborder sa complexité très en amont des projets de recherche. Dans cette étape préliminaire, peuvent aussi être associés à l'occasion, en plus des scientifiques, des personnes de toutes les communautés concernées. Mais les recherches qui se développeront seront très majoritairement disciplinaires, dites «éclairées », grâce à une problématisation amont commune et une indisciplinarité active.

Il n'y a pas, non plus, de coordination très forte $a$ priori. Les APR sont bien thématisés mais selon les axes larges de la problématique. Les interactions interviennent dans des occasions dédiées (séminaires, réunions...) ou plus ou moins fortuites (le travail simultané sur un même terrain provoque nécessairement des occasions d'échanges, notamment lors des moments de sociabilité). L'objet focal supprime des facteurs de blocage majeurs, responsables de nombreux échecs ${ }^{16}$. Il permet en effet de ne pas contraindre les différentes disciplines de travailler ensemble au départ de la recherche, de ne pas nécessiter une étroite coordination, de ne pas devoir imposer un cadre opérationnel commun à des temporalités et pratiques très souvent discordantes, ni d'avoir à identifier au préalable un «leader» reconnu par tous (Scholz et Steiner, 2015a et 2015b), de ne concevoir la visée intégrative que comme très générale pendant toute la durée des projets en ne la requérant précise et fonctionnelle qu'aux séminaires d'étapes et surtout à la

\footnotetext{
16 Pour un bel inventaire des difficultés rencontrées pour la mise en œuvre de la transdisciplinarité, $c f$. Scholz et Steiner, 2015b, tableau 1, p. 656-659.
}

fin, lors de la synthèse apportant explication et débouchant sur une modélisation.

Les exemples ci-dessus l'illustrent très concrètement, comme celui du mercure marin des lacs pyrénéens qui a emprunté le chemin de recherche suivant: géochimie: dosage des éléments traces $\rightarrow$ résultat inattendu : mercure marin $\rightarrow$ comment expliquer ? $\rightarrow$ que se passe-t-il dans les lacs : alevinage $\rightarrow$ étude des caractéristiques de l'alevinage (entretiens avec les acteurs et dépouillement des archives) $\rightarrow$ identification de l'origine du mercure marin $\rightarrow$ explication globale et identification des conséquences à venir $\rightarrow$ formulation de nouvelles questions : comment faire pour éviter cet apport contaminant? quelles sont les conséquences des pratiques de la pêche d'agrément? doiton et peut-on l'arrêter? etc.

Pour ce qui est de la transdisciplinarité, seule la voie «zurichoise», explicitement conçue pour résoudre matériellement des problèmes, pourrait nous concerner. Nous avons de fait beaucoup de notions en commun, mais notre pratique de recherche dans les OHM s'en distingue sur des points essentiels, certains également liés à l'organisation du travail et à ses temporalités, d'autres plus fondamentaux, notamment, ayant trait à l'échelle de l'objet de recherche et à la façon d'associer les acteurs sociaux.

Les questions qui nous concernent ne sont pas directement les «wicked problems» planétaires pointés par cette communauté (Brown et al., 2015; McGregor et Donelly, 2014). Nous nous positionnons, en entrée, à l'échelon local et sur des cas précis, comme l'illustrent ces deux nouveaux exemples. Dans le cas de l'OHM Estarreja (Portugal), le complexe chimique a déversé des quantités importantes de polluants métalliques et organiques à l'air libre jusque dans les années 1990. Où en est-on actuellement en termes de contaminations et de santé publique? Comment faire pour résoudre les problèmes qui y sont liés, s'il s'en rencontre? Dans l'OHM Tessékéré (Sénégal), quelles sont les conséquences de la construction de la Grande Muraille Verte (GMV), voulue par l'OUA pour ralentir l'aridification du Sahel, sur l'ESU de Tessékéré, socialement, écologiquement, économiquement, en termes de santé? Comment en valoriser ou accompagner les conséquences positives ou éventuellement limiter ou prévenir les négatives, en sachant que, dans tous les cas, l'apport de solutions ne relève pas de l'OHM, mais des personnes dont c'est socialement et politiquement la charge?

Nous n'incluons pas les acteurs politiques, sociaux, économiques, etc. dans le processus de recherche luimême car ils ne sont pas des chercheurs. L'expérience - celle des «Zurichois », comme la nôtre - montre qu'il est extrêmement difficile de faire participer de façon prolongée quelqu'un à une activité qui ne concerne ni ses compétences ni son statut (beaucoup d'OHM l'ont tenté, 
notamment dans leurs comités de pilotage). L'identification de l'objet d'un OHM est d'abord d'ordre scientifique. Pour le Bassin minier de Provence, par exemple, l'objet focal, travaillé par tous, est le territoire concerné par l'exploitation industrielle d'une mine de lignite pendant des décennies, puis affecté par sa fermeture et les conséquences sociales, économiques, écologiques qui en découlent. Ensuite, intervient la phase de coconstruction où chacun, depuis son cœur d'expertise, identifiera les points selon lui importants qui concernent cet objet, les questions qui se posent aujourd'hui et se poseront peut-être demain. Dans des occasions dédiées, des interactions seront organisées avec les acteurs non académiques du territoire pour identifier les expressions et questionnements sociétaux. La conversion des questions sociétales en questions de recherche relèvera cependant de la seule compétence des chercheurs. D'autres interactions interviendront pour optimiser les retombées de ces dispositifs. Les résultats de la recherche seront transmis dans des cadres scientifiques et aussi sociétaux. Il ne s'agit pas de construire un seul «nouveau savoir», mais de permettre l'enrichissement de chacun de la connaissance de ceux des autres, en tant que de pertinence. Au sein des OHM, les acteurs politiques, économiques et sociaux ne sont pas intégrés dans le processus de recherche, mais associés, à la place qui est celle de chacun et aux moments opportuns ${ }^{17}$.

Un des atouts des OHM est de poser la question des rapports entre les disciplines en amont des questions opérationnelles de recherche, dans un cadre clairement construit autour d'un objet focal partagé par tous et localement déterminé. C'est ce cadre préalable qui permet la mise en œuvre simple et aisée de la «disciplinarité éclairée» en levant la nécessité de coconstruction interdisciplinaire pour chaque projet de recherche, dispensant de cette étape sinon majeure mais lourde, fastidieuse (Wernli et Darbellay, 2016, p. 14), et grand facteur de blocage.

\section{Conclusion}

D'autres dispositifs se consacrent à l'étude de l'environnement, des écosystèmes ${ }^{18}$, certains des socioécosystèmes ${ }^{19}$ et à la mise en œuvre de l'interdisciplinarité (zones critiques ${ }^{20}$ TERENO [Bogena et al.,

\footnotetext{
17 Cette différence, fondamentale, est clairement exprimée aussi par Jacobs (2013, p. 128). On en trouvera une illustration très éloquente dans Scholz et Steiner $(2015 \mathrm{a}$, notamment p. 528-534).

18 sensu Tansley (1935).

19 sensu Gallopin (1991) ou Berkes et Folke (1998).

${ }^{20}$ sensu National Research Council (2001).
}

2016] et $\mathrm{OZCAR}^{21}$; écosystèmes et/ou socioécosystèmes LTER [Callahan, 1984; Redman et al., 2004], LTSER [Haberl et al., 2006; Ohl et al., 2010]; Zones Ateliers [ZA, Lévêque et al., 2000, 2008]; HERO [MacEachren et al., 2006; Yarnal et Neff, 2007]). Les OHM s'en distinguent fondamentalement par:

- un ciblage sur des objets très anthropisés, définis par des critères exclusivement anthropiques (crises);

- la considération que l'humain forme avec l'abiotique et le biologique un écosystème unique (ESU);

- leur conception avec fait structurant, événement fondateur et objet focal, organisation qui permet une prise en compte active de la complexité des systèmes par la mise en œuvre simple d'une interdisciplinarité efficace et disponible;

- la conception et la mise en auvre des interactions disciplinaires, avec l'indisciplinarité comme base et la «disciplinarité éclairée» comme résultat, avec des modalités spécifiques de l'interaction science-société, le tout constituant une interdisciplinarité caractéristique des OHM;

- la constitution d'un dispositif unique dont les composantes (les $\mathrm{OHM}$ ) sont en très fortes interactions. C'est ce que permet et représente le DRIIHM, ce métasystème qui dote tout le dispositif d'un fonctionnement en interactions et rétroactions, transcalaire, proprement écosystémique, bien plus puissant et libre qu'un simple réseau ${ }^{22}$.

Plus de 12 ans après la création des OHM et 7 ans d'existence du Labex DRIIHM, les exemples abondent pour montrer que le système mis en place fonctionne, qu'il permet, par la convergence d'apports disciplinaires ouverts et, par sa conception même, l'effacement de facteurs majeurs de blocages, d'arriver effectivement à la compréhension de systèmes complexes par la mise en œuvre d'une écologie globale. Tout aussi certain, cette caractéristique, jamais définitivement acquise, est le fruit d'une action et d'une vigilance permanentes. Le cercle vertueux doit être sans cesse entretenu. On peut espérer que cette attitude d'ouverture, malgré toutes les réticences individuelles et structurelles, s'ancre peu à peu dans les comportements. Cela semble progresser

\footnotetext{
${ }^{21} \mathrm{http}$ ://ozcar-ri.prod.lamp.cnrs.fr/ozcar/?lang=fr.

22 Dès la création des trois premiers OHM, la nécessité de développer entre eux des relations pour potentialiser les efforts réalisés et les résultats obtenus nous a conduits à identifier le réseau des OHM (ROHM). Nous avons depuis remarqué que la manière dont les OHM interagissaient entre eux ne caractérisait en rien un réseau mais quelque chose de bien plus complexe, un fonctionnement écosystémique, fait d'interactions et de rétroactions, transscalaire, permis par le DRIIHM. En 2019, nous en avons tiré les conséquences en supprimant la notion inadaptée de réseau de tout le dispositif du DRIIHM.
} 
dans la communauté des participants aux OHM, mais demeure aujourd'hui encore un travail de chaque jour. L'importante rotation des participants aux APR fait sans cesse arriver des personnes nouvelles, étrangères à ces fonctionnements et principes. C'est aussi dû à l'effet des modes de financements : faibles (pas plus de $10 \mathrm{~K} €$ ), mais aisés à obtenir (dossier léger et acceptation d'une prise de risques), de manière à fonder les bases sur lesquelles construire ensuite des projets beaucoup plus ambitieux à soumettre auprès d'autres sources de financement. Pour ancrer ces pratiques chez les participants, nous favorisons tout ce qui peut permettre cette imprégnation (projets pluriannuels, organisation d'occasions multiples d'échanges), faisons largement appel aux stagiaires de master (avec participation à tous les séminaires). Il est aussi prévu que nous nous coordonnions plus étroitement avec certaines universités pour faire percoler dans les cursus les principes de notre fonctionnement interdisciplinaire. L'enjeu est en fait de créer une communauté réceptive et accoutumée.

Enfin, jamais, au cours de ces années de fonctionnement, l'organisation en système disciplinaire de la recherche n'a semblé être un handicap, à partir du moment où chacun adoptait une attitude d'ouverture et avait à l'esprit que la compréhension globale du système étudié ne pouvait être atteinte sur la base de sa seule discipline, et cela uniquement à cause de la complexité et de l'importance des interactions/rétroactions qui s'y développaient. La position très pragmatique qui est la nôtre nous a dispensés de tout positionnement sur la postdisciplinarité. Jamais le désir «d'en finir avec les disciplines » (Darbellay, 2016, p. 363) n'a été rencontré. Notre expérience témoigne simplement de ce que les problèmes, pour nous, ne surgissent pas tant de la disciplinarité que de l'absence d'indisciplinarité.

\section{Références}

Apostel L., Berger G., Briggs A., Michaud G. (Eds), 1972. Interdisciplinarity. Problems of teaching and research in universities, Paris, OCDE.

Berkes F., Folke C. (Eds), 1998. Linking social and ecological systems. Management practices and social mechanisms for building resilience, Cambridge, Cambridge University Press.

Berkes F., Colding J., Folke C. (Eds), 2003. Navigating socialecological systems. Building resilience for complexity and change, Cambridge, Cambridge University Press.

Bernstein J.H., 2015. Transdisciplinarity: a review of its origins, development, and current issues, Journal of Research Practice, 11, 1, 1-17, http://jrp.icaap.org/index. php/jrp/article/view/510/412.

Bogena H., Borg E., Brauer A., Dietrich P., Hajnsek I., Heinrich I., Kiese R., Kunkel R., Kunstmann H., Merz B., Priesack E., Pütz T., Schmid H.P., Wollschläger U., Vereecken H., Zacharias S., 2016. TERENO: German network of terrestrial environmental observatories, Journal of Large-Scale Research Facilities, 2, 52, http://dx.doi.org/ 10.17815/j1srf-2-98.

Bourdieu P., 2001. Science de la science et réflexivité, Paris, Raisons d'agir.

Bourguignon A., 2014. De la pluridisciplinarité à la transdisciplinarité, http:/grit-transversales.org/archives/ revue/51/science1.html.

Brown R.R., Deletic A., Wong H.F., 2015. Interdisciplinarity: how to catalyse collaboration, Nature, 525, 315-317, https://doi.org/10.1038/525315a.

Callahan J.T., 1984. Long-Term Ecological Research, BioScience, 34, 6, 363-367, https://doi.org/10.2307/1309727.

Catellin S., Loty L., 2013. Sérendipité et indisciplinarité, Hermès, 67, 3, 32-40, https://doi.org/10.4267/2042/51882.

Chenorkian R., 2014. Éléments constitutifs des Observatoires hommes-milieux, origine et évolutions, in Chenorkian R., Robert S. (Eds), Les interactions hommes-milieux. Questions et pratiques de la recherche en environnement, Versailles, Quæ, 23-38, https://doi.org/10.3917/quae. cheno.2014.01.0023.

Chenorkian R., 2019. Changements, transitions et contextes écosystémiques très anthropisés, in Bedouret D. (Ed.), Actes $d u$ colloque "Changements et transitions: enjeux pour les éducations à l'environnement et au développement durable », Toulouse, 7-9 novembre 2017, https://doi.org/ 10.26147/geode.act.t1yg-gv72.

Chenorkian R., Abbadie L., 2017. Is resilience still relevant, in Euzen A., Laville B., Thiébault S. (Eds), Adapting to climate change, Paris, ediSens.

Darbellay F., 2011. Vers une théorie de l'interdisciplinarité? Entre unité et diversité, Nouvelles Perspectives en Sciences Sociales, 7, 1, 65-87, https://doi.org/10.7202/1007082ar.

Darbellay F., 2015. Rethinking inter- and transdisciplinarity: undisciplined knowledge and the emergence of a new thought style, Futures, 65, 163-174, https://doi.org/ 10.1016/j.futures.2014.10.009.

Darbellay F., 2016. From disciplinarity to postdisciplinarity: tourism studies dedisciplined, Tourism Analysis, 21, 4, 363372, https://doi.org/10.3727/108354216x14600320851659.

Darbellay F., 2018. L'interdisciplinarité, les aveugles et l'éléphant, Decentered Disciplines, https://decentered.hypo theses.org/1101.

Frodeman R., Thompson Klein J., Mitcham C. (Eds), 2010. The Oxford handbook of interdisciplinarity, Oxford, Oxford University Press.

Gallopin G.C., 1991. Human dimensions of global change: linking the global and the local processes, International Social Science Journal, 43, 4, 707-718.

Godard O., 1992. La relation interdisciplinaire : problèmes et stratégies, in Jollivet M. (Ed.), Sciences de la nature, sciences de la société. Les passeurs de frontières, Paris, CNRS Éditions, 427-456, https://doi.org/10.4000/books. editionscnrs.4210.

Graff H.J., 2015. Undiscipining knowledge, Baltimore, John Hopkins University Press.

Haberl H., Winiwarter V., Andersson K., Ayres R.U., Boone C., Castillo A., Cunfer G., Fischer-Kowalski M., Freudenburg 
W.R., Furman E., Kaufmann R., Krausmann F., Langthaler E., Lotze-Campen H., Mirtl M., Redman C.L., Reenberg A., Wardell A., Warr B., Zechmeister H., 2006. From LTER to LTSER: conceptualizing the socioeconomic dimension of Long-term socioecological research, Ecology and Society, 11, 2, 13. http://www.ecologyandsociety.org/vol11/iss2/art13/.

Jacobs J.A., 2013. In defense of disciplines. Interdisciplinarity and specialization in the research, Chicago, University of Chicago Press.

Jacobs J.A., Frickel S., 2009. Interdisciplinarity: a critical assessment, Annual Review of Sociology, 35, 43-65, https:// doi.org/10.1146/annurev-soc-070308-115954.

Jollivet M., 1992. Pluridisciplinarité, interdisciplinarité et recherche finalisée ou des rapports entre sciences, techniques et sociétés, in Jollivet M. (Ed.), Sciences de la nature, sciences de la société. Les passeurs de frontières, Paris, CNRS Éditions, 519-535, https://doi.org/10.4000/ books.editionscnrs. 4216 .

Kleinpeter E., 2013. Taxinomie critique de l'interdisciplinarité, Hermès, 67, 3, 123-129, https://doi.org/10.4267/2042/ 51898.

Lang D.J., Wiek A., Bergmann M., Stauffacher M., Martens P., Moll P., Swilling M., Thomas C.J., 2012. Transdisciplinary research in sustainability science: practice, principles, and challenges, Sustainability Science, 7, 1, 25-43, https://doi. org/10.1007/s11625-011-0149-x.

Lévêque C., Muxart T., Van Der Leeuw S., Weill A., Abbadie L., 2008. L'anthroposystème et la zone atelier : nouveaux concepts territorialisés de l'étude des interactions société/ milieux, in Alexandre F., Génin A. (Eds), Continu et discontinu dans l'espace géographique, Tours, Presses universitaires François Rabelais, 279-292, https://doi.org/ 10.4000/books.pufr.2383.

Lévêque C., Pavé A., Abbadie L., Weill A., Vivien F.-D., 2000. Les zones ateliers, des dispositifs pour la recherche sur l'environnement et les anthroposystèmes, Natures Sciences Sociétés, 8, 43-52, https://doi.org/10.1016/S1240-1307(01) 80005-4.

Loty L., 2005. Pour l'indisciplinarité, in Douthwaite J.V., Vidal M. (Eds), The indisciplinary century. Tensions and convergences in eighteen century art, history and literature, Oxford, Voltaire Foundation, 245-259, http://sciencesociete.fr/loty-laurent-pour-lindisciplinarite/.

MacEachren A.M., Pike W., Yu C., Brewer I., Gahegan M., Weaver S.D., Yarnal B., 2006. Building a geocollaboratory: supporting Human-Environment Regional Observatory (HERO) collaborative science activities, Computers, Environment and Urban Systems, 30, 201-225, https:// doi.org/10.1016/j.compenvurbsys.2005.10.005.

Max-Neef M.A., 2005. Foundations of transdisciplinarity, Ecological Economics, 53, 1, 5-16, https://doi.org/10.1016/ j.ecolecon.2005.01.014.

McGregor S.L.T., 2007. Consumer scholarship and transdisciplinarity, International Journal of Consumer Studies, 31, 487-495, https://doi.org/10.1111/j.1470-6431.2007.00599.x.

McGregor S.L.T., 2011. Place and transdisciplinarity, Transdisciplinary Journal of Engineering \& Science, 2, 10-19, https://doi.org/10.22545/2011/00020.
McGregor S.L.T., 2015. The Nicolescuian and Zurich approaches to transdisciplinarity, Integral Leadership Review, 15, 2, http://integralleadershipreview.com/13135-616-the-nicoles cuian-and-zurich-approaches-to-transdisciplinarity/.

McGregor S.L.T., Donelly G., 2014. Transleadership for transdisciplinary initiatives, World Futures, 70, 3-4, 164185, https://doi.org/10.1080/02604027.2014.934625.

McPhee C., Bliemel M., van der Bijl-Brouwer M., 2018. Editorial: transdisciplinary innovation, Technology Innovation Management Review, 8, 8, 3-6, http://doi.org/10.22215/ timreview/1173.

Moineau J.-C., 2015. Vive l'indisciplinarité ?, https://blog. transglobal-studies.org/vive-lindisciplinarite/.

Morin E., 1973. Le paradigme perdu: la nature humaine, Paris, Seuil.

Morin E., 1991. La Méthode. Les idées, t. 4, Paris, Seuil.

National Research Council, 2001. Basic research opportunities in Earth Science, Washington (DC), The National Academies Press, https://doi.org/10.17226/9981.

Nature, 2015. Interdisciplinarity, Nature, special issue, septembre, https://www.nature.com/collections/jcfdbccgjj.

Norman L.F., 2006. La transformation des disciplines dans l'université américaine, Cahiers de l'Association internationale des études francaises, 58, 21-34, https://doi.org/ 10.3406/caief.2006.1597.

Ohl C., Johst K., Meyerhoff J., Beckenkamp M., Grüsgen V., Drechsler M., 2010. Long-term socio-ecological research (LTSER) for biodiversity protection. A complex systems approach for the study of dynamic human-nature interactions, Ecological Complexity, 7, 170-178, https://doi.org/ 10.1016/j.ecocom.2009.10.002.

Peters D.P.C., Groffman P.M., Nadelhoffer K.J., Grimm N.B., Collins S.L., Michener W.K., Huston M.A., 2008. Living in an increasingly connected world: a framework for continental-scale environmental science, Frontiers in Ecology and the Environment, 6, 5, 229-237, https://doi.org/10.1890/ 070098.

Piaget J., 1972. L'épistémologie des relations interdisciplinaires, in Apostel L., Berger G., Briggs A., Michaud G. (Eds), L'interdisciplinarité. Problèmes d'enseignement et de recherche dans les universités, Paris, OCDE, 131-144, http://www.fondationjeanpiaget.ch/fjp/site/textes/VE/ jp72_epist_relat_interdis.pdf.

Redman C.L., Grove M.J., Kuby L.H., 2004. Integrating social science into the Long-term ecological research (LTER) network: social dimensions of ecological change and ecological dimensions of social change, Ecosystems, 7, 2, 161-171, https://doi.org/10.1007/s10021-003-0215-z.

Scholz R.W., Steiner G., 2015a. The real type and ideal type of transdisciplinary processes: part I-theoretical foundations, Sustainability Science, 10, 527-544, https://doi.org/ 10.1007/s11625-015-0326-4.

Scholz R.W., Steiner G., 2015b. The real type and ideal type of transdisciplinary processes: part II-what constraints and obstacles do we meet in practice?, Sustainability Science, 10, 653-671, https://doi.org/10.1007/s11625-015-0327-3.

Steffen W., Broadgate W., Deutsch L., Gaffney O., Ludwig C., 2015. The trajectory of the Anthropocene: the great 
acceleration, The Anthropocene Review, 2, 1, 81-98, https:// doi.org/10.1177/2053019614564785.

Tansley A.G., 1935. The use and abuse of vegetational concepts and terms, Ecology, 16, 3, 284-307, https://doi. org/10.2307/1930070.

Thompson Klein J., 2010. Une taxinomie de l'interdisciplinarité, Nouvelles Perspectives en Sciences Sociales, 7, 1, https://doi.org/10.7202/1007080ar.

Thompson Klein J., 2015. Reprint of "Discourses of transdisciplinarity: looking back to the future", Futures, 65, 10-16, https://doi.org/10.1016/j.futures.2015.01.003.
Tribe J., 1997. The indiscipline of tourism, Annals of Tourism Research, 24, 3, 638-657, https://doi.org/10.1016/ S0160-7383(97)00020-0.

Wernli D., Darbellay F., 2016. Interdisciplinarity and the 21st century research-intensive university, LERU, https://www. leru.org/publications/interdisciplinarity-and-the-21st-cen tury-research-intensive-university\#.

Yarnal B., Neff R., 2007. Teaching global change in local places: the HERO research experiences for undergraduates program, Journal of Geography in Higher Education, 31, 3, 413-426, https://doi.org/10.1080/03098260601082339.

Citation de l'article : Chenorkian R. Conception et mise en œuvre de l'interdisciplinarité dans les Observatoires hommesmilieux (OHM, CNRS). Nat. Sci. Soc. 28, 3-4, 278-291. 